\title{
INDEX TO VOLUME 75
}

\section{RESEARCH ANNOUNCEMENTS}

Aharonov, Dov. A note on slit mappings, 836.

Alexander, H. Uniform algebras on curves, 1269.

Allard, William K. On boundary regularity for Plateau's problem, 522.

Allegretto, W. and Swanson, C. A. Sturm comparison theorems for elliptic inequalities, 1318.

Alling, Norman and Greenleaf, Newcomb. Klein surfaces and real algebraic function fields, 869.

An, Chung-ming. On a generalization of the gamma function and its application to certain Dirichlet series, 562.

Anderson, R. D. Strongly negligible sets in Fréchet manifolds, 64.

Anderson, R. D. and Schori, R. A factor theorem for Frechet manifolds, 53.

Andrews, J. J. and Lomonaco, S. J. The second homotopy of spun 2-spheres in 4-space, 169.

Antonelli, P. L. On the stable diffeomorphism of homotopy spheres in the stable range, $n$ $<2 p, 343$.

Armentrout, Steve. Cellular decompositions of 3-manifolds that yield 3-manifolds, 453.

Arveson, William B. On subalgebras of $C^{*}$-algebras, 790.

Auslander, L. and Tolimieri, R. On a conjecture of G. D. Mostow and the structure of solvmanifolds, 1330.

Baily, Walter L., Jr. An exceptional arithmetic group and its Eisenstein series, 402.

Banks, H. T. A maximum principle for optimal control problems with functional differential systems, 158.

Ben-Israel, A., Charnes, A. and Kortanek, K. O. Duality and asymptotic solvability over cones, 318.

Berger, C. A. and Coburn, L. A. Erratum to: $C^{*}$-algebra of translations and multipliers, 468.

Berger, Melvyn S. On one parameter families of real solutions of nonlinear operator equations, 456.

Berndt, Bruce C. A note on the number of integral ideals of bounded norm in a quadratic number field, 1283.

Bojanic, R. and DeVore, R. A proof of Jackson's theorem, 364.

Boman, Jan and Shapiro, Harold S. Comparison theorems for a generalized modulus of continuity, 1266.

Brace, J. W. The space of continuous linear operators as a completion of $E^{\prime} \times F, 821$.

Breen, Lawrence S. On a nontrivial higher extension of representable abelian sheaves, 1249.

Brenner, J. L. Some inequalities from switching theory, 758.

Brillinger, David R. An asymptotic representation of the sample distribution function, 545.

Brooks, James K. and Reichelderfer, Paul V. On absolutely continuous transformations, 1243.

Brothers, John E. Rectifiability and integralgeometric measures in homogeneous spaces, 387.

Browder, Felix E. and Gupta, Chaitan P. Monotone operators and nonlinear integral equations of Hammerstein type, 1347.

Brown, H. I., Crawford, J. P. and Stratton, H. H. On summability fields of conservative operators, 992.

Brown, James R. A universal model for dynamical systems with quasi-discrete spectrum, 1028. 
Burgess, C. E. A characterization of homogeneous plane continua that are circularly chainable, 1354.

Charnes, A. See Ben-Israel, A.

Chen, Kuo-Tsai. An algebraic dualization of fundamental groups, 1020.

Cheney, E. W., Hobby, C. R., Morris, P. D., Schurer, F. and Wulbert, D. E. On the minimal property of the Fourier projection, 51.

Chow, T. R. See Goldstein, Myron.

Coburn, L. A. See Berger, C. A.

Coffman, C. V. and Wong, J. S. W. Second order nonlinear oscillations, 1379.

Cohen, Joel M. and Gluck, Herman. Stacked bases for modules, 978.

Collins, H. S. See Porcelli, Pasquale.

Colton, David and Gilbert, Robert P. Function theoretic methods in the theory of boundary value problems for generalized metaharmonic functions, 948.

Conlon, Lawrence. Applications of affine root systems to the theory of symmetric spaces, 610.

Cordes, H. O. An algebra of singular integral operators with two symbol homomorphisms, 37.

Corner, A. L. S. Additive categories and a theorem of W. G. Leavitt, 78.

Corwin, Lawrence. A "functional equation" for measures and a generalization of Gaussian measures, 829.

Cossey, John, Macdonald, Sheila Oates and Street, Anne Penfold. On the laws of certain linear groups, 361.

Crawford, J. P. See Brown, H. I.

Crittenden, Richard B. and Vanden Eynden, C. L. A proof of a conjecture of Erdös, 1326.

Curtis, Edward B. Some nonzero homotopy groups of spheres, 541.

Davis, Chandler and Kahan, W. M. Some new bounds on perturbation of subspaces, 863.

Davis, R. L. A Galois theory for a class of purely inseparable exponent two field extensions, 1001.

Della Riccia, Giacomo. Iteration of analytic mappings of several complex variables, 340 .

DeSapio, R. Manifolds homeomorphic to sphere bundles over spheres, 59.

Deutsch, Frank and Morris, Peter D. On simultaneous approximation and interpolation which preserves the norm, 812.

DeVore, R. See Bojanic, R.

Diaz, J. B. and McLaughlin, Joyce R. Sturm comparison theorems for ordinary and partial differential equations, 335.

Dotson, W. G. Jr. An application of ergodic theory to the solutions of linear functional equations in Banach spaces, 347.

Drasin, David and Shea, Daniel F. Asymptotic properties of entire functions extremal for the cos $\pi \rho$ theorem, 119.

Dress, Andreas. On relative Grothendieck rings, 955.

On the decomposition of modules, 984. . On integral representations, 1031.

Duran, Peter L. Extension of a theorem of Carleson, 143.

Eaton, W. T. Cross sectionally simple spheres, 375.

Ebin, David G. and Marsden, Jerrold E. Groups of diffeomorphisms and the solution of the classical Euler equations for a perfect fluid, 962.

Essén, Matts. A generalization of the Ahlfors-Heins theorem, 127.

Faulkner, John R. Octonion planes in characteristic two, 980.

Fearnley, Lawrence. The pseudo-circle is unique, 398.

-. The pseudo-circle is not homogeneous, 554. 
Fefferman, Charles. On some singular convolution operators, 765.

Fennell, Robert, and Waltman, Paul. Boundary value problems for functional differential equations, 487.

Fink, A. M. Compact families of almost periodic functions, 770.

Fisher, Stephen. Another theorem on convex combinations of unimodular functions, 1037.

Flanders, Harley. Free exterior powers, 505.

Fugita, Hiroshi. On the nonlinear equations $\Delta u+e^{u}=0$ and $\partial v / \partial t=\Delta v+e^{v}, 132$.

Fujita, Hiroshi and Sauer, Niko. Construction of weak solutions of the Navier-Stokes equation in a noncylindrical domain, 465.

Fulton, William. A note on weakly complete algebras, 591.

Garland, Howard. The spectrum of noncompact $G / \Gamma$ and the cohomology of arithmetic groups, 807.

Giffen, Charles H. Desuspendability of free involutions on Brieskorn spheres, 426.

- Smooth homotopy projective spaces, 509.

Gilbert, Robert P. A method of ascent for solving boundary value problems, 1286.

See Colton, David.

Glasner, Moses and Katz, Richard. A note on the Royden boundary, 945.

Gluck, Herman. Piecewise linear groups and transformation groups, 407.

- See Cohen, Joel M.

Goguen, J. A. Categories of V-sets, 622.

Goldstein, Charles. Eigenfunction expansions and similarity for certain nonselfadjoint operators, 550.

Goldstein, Myron and Chow, T. R. A theorem of Stout, 953.

von Golitschek, M. Generalization of the Jackson approximation theorems in the sense of Ch. Müntz, 524.

Goodman, Gerald S. A method for comparing univalent functions, 517.

Gordon, S. Robert. On the automorphism group of a semisimple Jordan algebra of characteristic zero, 499.

Graham, R. L. and Rothschild, B. L. Ramsey's theorem for n-dimensional arrays, 418.

Gray, Neil. $A$ new characterization of $A R(\mathfrak{T C})$ and $A N R(\mathfrak{T}), 607$.

Greene, Robert E. Isometric embeddings, 1308.

Greenleaf, Newcomb. See Alling, Norman.

Groetsch, C. W. See Outlaw, Curtis.

Grossberg, Stephen. Learning and energy-entropy dependence in some nonlinear functional-differential systems, 1238.

Gupta, Chaitan P. See Browder, Felix B.

Gustafson, Karl. On projections of selfadjoint operators and operator product adjoints, 739.

Hall, P. R. On the space of homomorphisms of $E^{3}, 788$.

Hallam, T. G. and Heidel, J. W. The asymptotic manifold of a nonlinear system of differential equations, 1290.

Heidel, J. W. See Hallam, T. G.

Heil, Wolfgang. On $P^{2}$-irreducible 3-manifolds, 772.

Helfenstein, Heinz G. Analytic maps between tori, 857.

Henderson, David W. Infinite-dimensional manifolds are open subsets of Hilbert space, 759.

Hertzig, David. Cohomology of certain Steinberg groups, 35.

Hill, Paul. A countability condition for primary groups presented by relations of length two, 780.

Hirsch, Morris W. and Pugh, Charles C. Stable manifolds for hyperbolic sets, 149. 
Hobby, C. R. See Cheney, E. W.

Hofmann, Karl Heinrich and Mostert, Paul. S. About the cohomology ring of a finite abelian group, 391.

Holland, John H. A new kind of turnpike theorem, 1311.

Hollingsworth, John. Triangulating nonsimply connected manifolds, 833.

Hosay, Norman. A proof on the slicing theorem for 2-spheres, 370.

Husch, L. S. Mapping cylinders and the annulus conjecture, 506.

Jaco, William. Three-manifolds with fundamental group a free product, 972.

James, I. M. On sphere-bundles. I, 617.

James, R. C. See Retherford, J. R.

Jarden, Moshe. Rational points on algebraic varieties over large number fields, 603.

Jategaonkar, Arun Vinayak. Local left noetherian ipli-rings, 514.

. Rings with transfinite left division algorithm, 559.

- Order in Artinian rings, 1258.

Jenkins, Joe W. An amenable group with a nonsymmetric group algebra, 357.

Johnson, J. A. Banach spaces of Lipschitz functions and vector-valued Lipschitz functions, 1334.

Jung, Calvin F. K. On generalized complete metric spaces, 113.

Kahan, W. M. See Davis, Chandler.

Kahn, Daniel S. Squaring operations in the Adams spectral sequence, 136.

Kato, Mitsuyoshi. Relative Hauptvermutung for neighborhoods of 1-flat submanifolds with codimension two, 1006.

- Embedding spheres and balls in codimension $\leqq 2,1260$.

Katz, Richard. See Glasner, Moses.

Kesten, Harry. A convolution equation and hitting probability of single points for processes with stationary independent increments, 573.

Kirby R. C. and Siebenmann, L. C. On the triangulation of manifolds and the Hauptvermutung, 742.

Kleinfeld, Erwin, Kleinfeld, Margaret Humm and Kosier, J. Frank. The structure of generalized accessible rings, 415.

Kleinfeld, Margaret Humm. See Kleinfeld, Erwin.

Kortanek, K. O. See Ben-Israel, A.

Kosier, Frank. See Kleinfeld, Erwin.

Kostant, Bertram and Rallis, Steven. On orbits associated with symmetric spaces, 879.

- On representations associated with symmetric spaces, 884.

Kotlow, Daniel B. On the equations $u_{t}+\nabla \cdot F(u)=0$ and $u_{t}+\nabla \cdot F(u)=\nu \Delta u, 1362$.

Kreith, Kurt. A Sturm theorem for strongly elliptic systems and applications, 1025.

Kujala, Robert O. Functions of finite $\lambda$-type in several complex variables, 104.

Kulkarni, Ravindra S. Curvature structures and conformal transformations, 91.

Lam, T. Y. and Reiner, I. Relative Grothendieck rings, 496.

Lashof, R. Lee's immersion theorem and the triangulation of manifolds, 535.

Lashof, R. and Rothenberg, M. Triangulation of manifolds. I, 750.

- Triangulation of manifolds, II, 755.

Lashof, Richard K. and Shaneson, Julius L. Classification of knots in codimension two, 171.

Lazarov, Connor and Wasserman, Arthur. Cobordism of regular $O(n)$-manifolds, 1365.

Lebow, Arnold and Schechter, Martin. Norms on quotient spaces, 1322.

Lee, Keean. $A$ note of functors ext over the ring $Z, 852$.

Lee, Ronnie. Differentiable classification of some topologically linear actions, 441. 
Lees, J. Alexander. Immersions and surgeries of topological manifolds, 529.

Lin, Sue-Chin. On smoothness of gentle perturbations, 445.

Little, John A. On singularities of surfaces in $E^{4}, 368$.

Littman, Walter. Maximal rates of decay of solutions of partial differential equations, 1273.

Lomonaco, S. J. See Andrews, J. J.

Loveland, L. D. Cross sectionally continuous spheres in $E^{3}, 396$.

Macdonald, Sheila Oates. See Cossey, John.

Marcus, Marvin. Spectral properties of higher derivations on symmetry classes of tensors, 1303.

Marsden, Jerrold E. See Ebin, David G.

Mattuck, Arthur. Ruled surfaces and the Albanese mapping, 776.

McAuley, Louis F. Certain mappings or decompositions which are topologically projections, 941.

McEliece, Robert J. and Rumsey, Howard, Jr. Sphere-packing in the Hamming metric, 32.

McLaughlin, Joyce R. See Diaz, J. B.

Mizel, V. J. Representations of nonlinear transformations on $L^{p}$ spaces, 164.

Montgomery, M. Susan. Left and right inverses in group algebras, 539.

Moore, Robert T. Banach algebras of operators on locally convex spaces, 68.

- Adjoints, numerical ranges, and spectra of operators on locally convex spaces, 85.

Morawetz, Cathleen S. Two $L^{p}$ inequalities, 1299.

Morris, P. D. See Cheney, E. W.

Morris, Peter D. See Deutsch, Frank.

Mostert, Paul S. See Hofmann, Karl Heinrich.

Muhly, Paul S. Commutants containing a compact operator, 353.

Newman, Stephen E. Measure algebras and functions of bounded variation on idempotent semigroups, 1396.

Nielsen, Ole A. Maximal abelian subalgebras in hyperfinite factors, 579.

Nitsche, Johannes C. C. A variational problem with inequalities as boundary conditions, 450.

Nussbaum, Roger D. The fixed point index and asymptotic fixed point theorems for $k$ set-contractions, 490.

Oberst, Ulrich. Duality theory for Grothendieck categories, 1401.

O'Neil, Patrick Eugene. Asymptotics and random matrices with row-sum and column sum-restrictions, 1276.

Ono, Takashi. On Gaussian sums, 43.

Osserman, Robert. The nonexistence of branch points in the classical solution of Plateau's problem, 1247.

Outlaw, Curtis and Groetsch, C. W. Averaging iteration in a Banach space, 430.

Palais, Richard S. The Morse lemma for Banach spaces, 968.

Pfaltzgraff, J. A. and Pinchuk, Bernard. Constrained extremal problems for classes of meromorphic functions, 379.

Pfeffer, W. F. An integral in topological spaces, 433.

Phillips, Keith. Maximal functions for a class of locally compact noncompact groups, 384.

Pinchuk, Bernard. See Pfaltzgraff, J. A.

Pless, Vera. On a new family of symmetry codes and related new five-designs, 1339.

Porcelli, Pasquale and Collins, H. S. Ideals in group algebras, 83.

Port, Sidney C. and Stone, Charles J. Potential theory for infinitely divisible processes on Abelian groups, 848. 
Porta, Horacio. Two sided ideals of operators, 599.

Prenter, P. M. A Weierstrass theorem for normed linear spaces, 860.

Pugh, Charles C. See Hirsch, Morris W.

Quillen, Daniel. On the formal group laws of unoriented and complex cobordism theory, 1293.

Rallis, Steven. See Kostant, Bertram.

Rao, B. V. On discrete Borel spaces and projective sets, 614.

Rao, M. M. Contractive projections and prediction operators, 1369.

Rao, V. V. L. N. On properties of self reciprocal functions, 162.

Reichelderfer, Paul V. See Brooks, James K.

Reiner, I. See Lam, T. Y.

Retherford, J. R. and James R. C. Unconditional bases and best approximation in Banach spaces, 108.

Rieger, G. J. On polynomials and almost-primes, 100.

Rivière, N. M. On singular integrals, 843.

Roberts, A. Wayne and Varberg, Dale E. Functions of bounded convexity, 568.

Robertson, Lewis C. A note on the structure of Moore groups, 594.

Rockafellar, R. T. Convexity properties of nonlinear maximal monotone operators, 74.

Rosenblum, Marvin and Rovnyak, James. Factorization of operator valued entire functions, 1343.

Rosenthal, Haskell P. Some linear topological properties of $L^{\infty}$ of a finite measure space, 798.

- On injective Banach spaces and the spaces $C(S), 824$.

Rota, Gian-Carlo. Baxter algebras and combinatorial identities. I, 325.

. Baxter algebras and combinatorial identities. II, 330.

Rothenberg, M. See Lashof, R.

Rothschild, B. L. See Graham, R. L.

Rovnyak, James. See Rosenblum, Marvin.

Rudin, Walter. Convex combinations of unimodular functions, 795.

Rumsey, Howard, Jr. See McEliece, Robert J.

Rushing, T. B. Taming codimension three embeddings, 815.

Sard, Arthur. A theory of cotypes, 936.

Sahney, Badri N. A note on matrix summability of a class of Fourier series, 1374.

Sauer, Niko. See Fujita, Hiroshi.

Schechter, Martin. Partial differential operators on $L^{p}\left(E^{n}\right), 548$.

- See Lebow, Arnold.

Schonefeld, Steven. Schauder bases in spaces of differentiable functions, 586.

Schori, R. See Anderson, R. D.

Schurer, F. See Cheney, E. W.

Schurle, Arlo W. Decompositions of $E^{3}$ into points and countably many trees, 422.

Segal, Irving. Notes towards the construction of nonlinear relativistic quantum fields.

II: The basic nonlinear functions in general space-times, 1383.

- Notes towards the construction of nonlinear relativistic quantum fields. III:

Properties of the $C^{*}$-dynamics for a certain class of interactions, 1390.

Shah, S. M. and Trimble, S. Y. Univalent functions with univalent derivatives, 153, 888.

Shaneson, Julius L. See Lashof, Richard K.

Shapiro, Harold S. See Boman, Jan.

Shea, Daniel F. See Drasin, David.

Shub, Michael. Periodic orbits of hyperbolic diffeomorphisms and flows, 57.

Siebenmann, L. C. See Kirby, R. C.

Siu, Yum-Tong. Extending coherent analytic sheaves through subvarities, 123, 624. 
Analytic sheaves of local cohomology, 1011.

Small, Lance W. The embedding problem for Noetherian rings, 147.

Smith, J. Wolfgang. Commuting vectorfields on open manifolds, 1013.

Smith, Larry. On the construction of the Eilenberg-Moore spectral sequence, 873.

Stampfli, J. G. A local spectral theory for operators. II, 803.

Stasheff, James D. Manifolds of the homotopy type of (non-Lie) groups, 998.

Stein, James D., Jr. On generalized complete metric spaces, 439.

Stone, Charles J. See Port, Sidney C.

Stratton, H. H. See Brown, H. I.

Street, Anne Penfold. See Cossey, John.

Summers, W. H. Products of fully complete spaces, 1005.

Swanson, C. A. See Allegretto, W.

Szczarba, R. H. On existence and rigidity of isometric immersions, 783.

Tolimieri, R. See Auslander, L.

Trimble, S. Y. See Shah, S. M.

Uhl, J. J., Jr. Applications of Radon-Nikodym theorems to martingales of vector valued functions, 840.

Vanden Eryden, C. L. See Crittenden, Richard B.

Varberg, Dale E. See Roberts, A. Wayne.

Veech, William A. Minimal transformation groups with distal points, 481.

Vick, James W. An application of K-theory to equivariant maps, 1017.

Walker, Leroy H. Regarding stopping rules for Brownian motion and random walks, 46.

Wallen, Lawrence J. Semigroups of partial isometries, 763.

Waltman, Paul. See Fennell, Robert.

Wasserman, Arthur. See Lazarov, Connor.

Waterman, Daniel. W-systems are the Walsh functions, 139.

Weber, Hans R. Canonical theory of the nonparametric Lagrangian multiple integral problems with variable boundaries, 460 .

Weinstein, Alan. Symplectic structures on Banach manifolds, 1040.

Wells, John. Differentiable functions on $c_{0}, 117$.

West, James E. Approximating homotopies by isotopies in Fréchet manifolds, 1254.

Williams, David. Markov properties of Brownian local time, 1035.

Williams, R. E. A note on rings with a weak algorithm, 959.

Williamson, Robert. Surgery in $M \times N$ with $\pi_{1} M \neq 1,582$.

Wilson, Robert Lee. Nonclassical simple Lie algebras, 987.

Wogen, Warren. On generators for von Neumann algebras, 95.

Wong, J. S. W. See Coffman, C. V.

Wong, Yung-Chow. Euclidean n-planes in pseudo-Euclidean spaces and differential geometry of Cartan domains, 409.

Wu, H. An n-dimensional extension of Picard's theorem, 1357.

Wulbert, D. E. See Cheney, E. W.

\section{BOOK REVIEWS}

Almgren, F. J., Jr. Plateau's problem an invitation to varifold geometry. William P. Ziemer, 924.

Bailey, Paul, Shampine, Lawrence and Waltman, Paul. Nonlinear two point boundary value problems. J. W. Bebernes, 724.

Blumenthal, R. M. and Getoor, R. K. Markov processes and potential theory. P. A. Meyer, 912.

Breiman, Leo. Probability. Louis Sucheston, 706. 
Chung, Kai Lai. A course in probability. Louis Sucheston, 706.

Getoor, R. K. See Blumenthal, R. M.

Halmos, Paul R. A Hilbert space problem book. Joseph G. Stampfli, 923.

Herstein, I. N. Noncommutative rings. Maria J. Wonenburger, 714.

Katznelson, Yitzhak. An introduction to harmonic analysis. J. Ian Richards, 709.

Ladyženskaja, O. A. and Ural'ceva, N. N. Linear and quasilinear elliptic equations.

N. S. Trudinger, 720.

Lang, Serge. Rapport sur la cohomologie des groupes. G. Hochschild, 927.

Miller, Willard, Jr. Lie theory and special functions. Louis Weisner, 925.

Mosher, Robert E. and Tangora, Martin C. Cohomology operations and applications in homotopy theory. M. Mahowald, 929.

Noshiro, Kiyoshi. See Sario, Leo.

Pierce, R. S. Introduction to the theory of abstract algebras. P. M. Cohn, 717.

Sario, Leo and Noshiro, Kiyoshi. Value distribution theory. James A. Jenkins, 917.

Schafer, R. D. An introduction to nonassociative algebras. Maria J. Wonenburger, 712.

Shampine, Lawrence. See Bailey, Paul.

Spanier, Edwin H. Algebraic topology. F. P. Peterson, 916.

Tangora, Martin C. See Mosher, Robert E.

Ural'ceva, N. N. See Ladyženskaja, O. A.

Valentine, F. A. Convex sets. Victor Klee, 722.

Waltman, Paul. See Bailey, Paul.

\section{REPORTS OF MEETINGS AND MISCELLANEOUS ARTICLES}

Bateman, Paul T. The November Meeting in Evanston, 317; The April Meeting in Cincinnati, 930.

- See Pitcher, Everett.

Berger, C. A. and Coburn, L. A. Erratum to: $C^{*}$-algebras of translations and multipliers, 468.

Coburn, L. A. See Berger, C. A.

Council and Board of Trustees-1968, 176.

Federer, Herbert. The October Meeting in Baltimore, 28.

Gillman, Leonard. See Pitcher, Everett.

Harrold, O. G. The November Meeting in Clemson, 29. See Pitcher, Everett.

Martin, W. T. Report of the Treasurer-1968, 933.

Pierce, R. S. The November Meeting in Riverside, 30; The April meeting in Santa Cruz, 932.

Pitcher, Everett. 1968 Election of Members of the Council and Board of Trustees, 31.

Pitcher, Everett and Bateman, Paul T. The Summer Meeting in Madison, 11.

Pitcher, Everett and Gillman, Leonard. The April Meeting in New York, 728.

Pitcher, Everett and Harrold, O. G. The Annual Meeting in New Orleans, 471.

Spencer, D. C. Overdetermined systems of linear partial differential equations, 179.

Thom, R. Ensembles et morphismes stratifiés, 240.

Wilder, R. L. Trends and social implications of research, 891.

\section{INVITED ADDRESSES}

Almgren, F. J., Jr. Measure theoretic geometry and elliptic variational problems, 285.

Boone, William W., 930.

Browder, William, 728. 
Douglis, Avron, 28.

Edwards, C. H., Jr., 29.

Fadell, Edward R., 930.

Fuchs, Laszlo, 29.

Ganea, Theodor, 932.

Griffith, Phillip A., 11.

Guillemin, Victor W., 11.

Harrison, David, 932.

Higman, Donald G., 317.

Hsiang, Wu-Chung, 11.

Hsiang, Wu-Yi, 317.

Kostant, Bertram. On the existence and irreducibility of certain series of representations, 627.

Magnus, Wilhelm, 28.

. Residually finite groups, 305.

Masani, Pesi R., 930.

McKean, H. P., Jr. A simple model of the derivation of fluid mechanics from the Boltzmann equation, 1.

Milnor, John W., 11.

Moore, Calvin C., 471.

Morawetz, Cathleen, 728.

Morrey, Charles B., Jr. Differentiability theorems for weak solutions of nonlinear elliptic differential equations, 684.

Moser, Jürgen, K., 471.

Nomizu, Katsumi, 728.

Osserman, Robert. Minimal varieties, 1092.

- 30.

Peterson, Franklin P. Characteristic classes-old and new, 907.

Robertson, Malcolm, 29.

Shale, David, 728.

Spencer, Donald C., 11.

Suzuki, Michio. Characterizations of linear groups, 1043.

Thomas, Emery. Vector fields on manifolds, 643.

Treves, François, 930.

Wilder, R. L., 471.

Wolf, Joseph, 30.

. The action of a real orbit semisimple group on a complex flag manifold. I: Orbit structure and holomorphic arc components, 1121. 